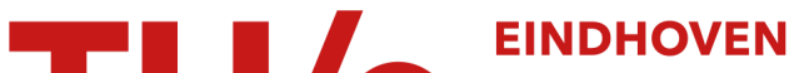 UNIVERSITY OF TECHNOLOGY
}

\section{Graceful degradation of cooperative adaptive cruise control}

Citation for published version (APA):

Ploeg, J., Semsar-Kazerooni, E., Lijster, G., Wouw, van de, N., \& Nijmeijer, H. (2015). Graceful degradation of cooperative adaptive cruise control. IEEE Transactions on Intelligent Transportation Systems, 16(1), 488-497. https://doi.org/10.1109/TITS.2014.2349498

DOI:

10.1109/TITS.2014.2349498

Document status and date:

Published: 01/01/2015

\section{Document Version:}

Publisher's PDF, also known as Version of Record (includes final page, issue and volume numbers)

\section{Please check the document version of this publication:}

- A submitted manuscript is the version of the article upon submission and before peer-review. There can be important differences between the submitted version and the official published version of record. People interested in the research are advised to contact the author for the final version of the publication, or visit the $\mathrm{DOI}$ to the publisher's website.

- The final author version and the galley proof are versions of the publication after peer review.

- The final published version features the final layout of the paper including the volume, issue and page numbers.

Link to publication

\section{General rights}

Copyright and moral rights for the publications made accessible in the public portal are retained by the authors and/or other copyright owners and it is a condition of accessing publications that users recognise and abide by the legal requirements associated with these rights.

- Users may download and print one copy of any publication from the public portal for the purpose of private study or research.

- You may not further distribute the material or use it for any profit-making activity or commercial gain

- You may freely distribute the URL identifying the publication in the public portal.

If the publication is distributed under the terms of Article $25 \mathrm{fa}$ of the Dutch Copyright Act, indicated by the "Taverne" license above, please follow below link for the End User Agreement:

www.tue.nl/taverne

Take down policy

If you believe that this document breaches copyright please contact us at:

openaccess@tue.nl

providing details and we will investigate your claim. 


\title{
Graceful Degradation of Cooperative Adaptive Cruise Control
}

\author{
Jeroen Ploeg, Elham Semsar-Kazerooni, Member, IEEE, Guido Lijster, \\ Nathan van de Wouw, and Henk Nijmeijer, Fellow, IEEE
}

\begin{abstract}
Cooperative adaptive cruise control (CACC) employs wireless intervehicle communication, in addition to onboard sensors, to obtain string-stable vehicle-following behavior at small intervehicle distances. As a consequence, however, CACC is vulnerable to communication impairments such as latency and packet loss. In the latter case, it would effectively degrade to conventional adaptive cruise control (ACC), thereby increasing the minimal intervehicle distance needed for string-stable behavior. To partially maintain the favorable string stability properties of CACC, a control strategy for graceful degradation of one-vehicle look-ahead CACC is proposed, based on estimating the preceding vehicle's acceleration using onboard sensors, such that the CACC can switch to this strategy in case of persistent packet loss. In addition, a switching criterion is proposed in the case that the wireless link exhibits increased latency but does not (yet) suffer from persistent packet loss. It is shown through simulations and experiments that the proposed strategy results in a noticeable improvement of string stability characteristics, when compared with the ACC fallback scenario.
\end{abstract}

Index Terms-Cooperative adaptive cruise control (CACC), graceful degradation, string stability, vehicle platoons, wireless communications.

\section{INTRODUCTION}

$\mathbf{C}$ OOPERATIVE adaptive cruise control (CACC) is a vehicle-following control system that automatically accelerates and decelerates so as to keep a desired distance from the preceding vehicle [1]. To this end, onboard sensors are employed, such as radar, which measure the intervehicle distance and relative velocity. In addition, information of the preceding vehicle(s), e.g., their intended acceleration, is cast through a wireless link. As a result, the performance in terms of minimizing the intervehicle distance while guaranteeing string stability, i.e., shockwave attenuation in upstream direction [2], is significantly enhanced when compared with

Manuscript received January 31, 2014; revised July 7, 2014; accepted August 11, 2014. Date of publication September 23, 2014; date of current version January 30, 2015. This work was supported by the European Union Seventh Framework Program (FP7/2007-2013) under Grant Agreement 257462 HYCON2 Network of Excellence. The Associate Editor for this paper was B. de Schutter.

J. Ploeg and E. Semsar-Kazerooni are with the Netherlands Organization for Applied Scientific Research TNO, 5700 AT Helmond, The Netherlands (e-mail: jeroen.ploeg@tno.nl; elham.semsarkazerooni@tno.nl).

G. Lijster is with TSCC Technology, 5657 EA Eindhoven, The Netherlands (e-mail: guido.lijster@tscc.nl).

N. van de Wouw and H. Nijmeijer are with the Department of Mechanical Engineering, Eindhoven University of Technology, 5612 AZ Eindhoven, The Netherlands (e-mail: n.v.d.wouw@tue.nl; h.nijmeijer@tue.nl).

Color versions of one or more of the figures in this paper are available online at http://ieeexplore.iee.org.

Digital Object Identifier 10.1109/TITS.2014.2349498 conventional adaptive cruise control (ACC), which is operated without wireless communication [3]. As a result, traffic throughput is increased, while maintaining safety [4].

Inherent in the CACC concept is its vulnerability to latency and packet loss of the wireless link, which will inevitably occur with an increasing amount of communicating vehicles employing the same network. The effect of latency of the wireless link on string stability in a vehicle platoon already attracted quite some attention in the past. In [5], for instance, a minimum string-stable time gap was derived as a linear function of the latency for a one-vehicle look-ahead control scheme, whereas [6] investigated the effect of communication latency on string stability with a controller that uses lead vehicle information in addition to preceding vehicle information. More recently, [7] focused on the relation between communication delay, controller parameters, and string stability for single- and multiplevehicle look-ahead communication topologies. Furthermore, [8] proposed an analysis framework incorporating uncertain sampling intervals and delays. Next to latency, packet loss is also of major importance. In [9], for instance, it was found that the ratio of correctly received packets drops to values below $10 \%$ on a motorway junction with high traffic density, assuming all vehicles are equipped with wireless communication devices. Taking packet loss into account, [10] focused on $H_{\infty}$ controller synthesis, whereas the experimental study described in [11] analyzed the effects on string stability for a given controller.

In contrast to the aforementioned literature, the main focus in this paper is on how to cope with losing the wireless link for an extended period of time. In this case, while not taking any compensating actions, CACC inherently degrades to ACC, which requires a significantly larger time gap to guarantee string-stable behavior. As an example, [12] shows that the minimum string-stable time gap increases from $0.7 \mathrm{~s}$ to more than $3 \mathrm{~s}$. To provide a certain level of fault tolerance against this type of wireless communication failures, a fallback strategy is presented to gracefully degrade functionality of a one-vehicle look-ahead CACC in the sense that a less dramatic increase in time gap is required to regain string-stable behavior. This strategy employs estimation of the preceding vehicle's acceleration using the available data from an onboard sensor. Using an experimental setup of three CACC-equipped passenger cars, the theoretical results are validated against measurements. In addition, a criterion is proposed to switch to this fallback strategy in the case that the wireless link is not (yet) completely lost, but shows a relatively large latency.

This paper is organized as follows. Section II introduces the notion of string stability as used in this paper and presents 


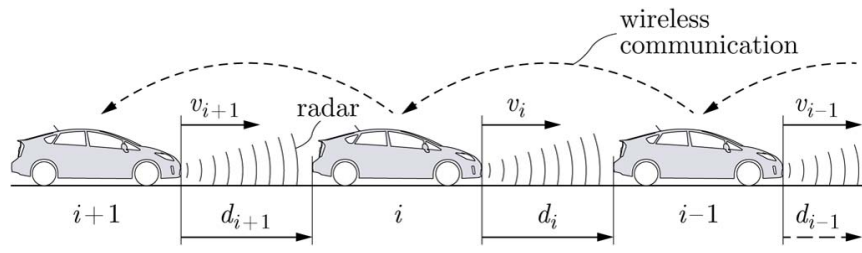

Fig. 1. Homogeneous platoon of vehicles equipped with CACC.

the nominal CACC strategy. Next, Section III introduces the graceful degradation strategy, upon which Section IV analyzes the string stability properties of the controlled system. Section $\mathrm{V}$ proposes a criterion for switching to degraded mode, after which Section VI presents experimental results. Finally, Section VII summarizes the main conclusions.

\section{Control of Vehicle Platoons}

Consider a homogeneous platoon of $m$ vehicles, as shown in Fig. 1, where the vehicles are enumerated with index $i=$ $1, \ldots, m$, with $i=1$ indicating the lead vehicle. To maintain a short intervehicle distance $d_{i}$ while not compromising safety, an automatic controller is required, which regulates $d_{i}$ to the desired value. An important requirement for this controller is to realize string-stable behavior of the platoon, which refers to the attenuation along the platoon of the effects of disturbances caused by, e.g., sudden braking of the lead vehicle. This section formally introduces the notion of string stability and describes the CACC controller, which is the basis for the graceful degradation strategy as presented in Section III.

\section{A. String Stability of a Vehicle Platoon}

In the literature, three main directions toward defining string stability can be distinguished: 1) a Lyapunov-stability approach [13]; 2) a stability approach for spatially invariant linear systems [14]; and 3) a frequency-domain approach [1], [15]. In [16], an overview of relevant literature on this topic is given, based on which string stability conditions for linear unidirectionally coupled homogeneous systems are formulated, similar to those used in the frequency-domain approach. These conditions are summarized hereafter.

Let the homogeneous vehicle platoon, in which all follower vehicles are controlled by a one-vehicle look-ahead CACC, be formulated in the state space as (omitting the time argument $t$ )

$$
\dot{x}=A x+B u_{1}
$$

with

$$
A=\left(\begin{array}{cccc}
A_{0} & & & \mathrm{O} \\
\tilde{A}_{1} & \tilde{A}_{0} & & \\
& \ddots & \ddots & \\
\mathrm{O} & & \tilde{A}_{1} & \tilde{A}_{0}
\end{array}\right), \quad B=\left(\begin{array}{c}
B_{0} \\
0 \\
\vdots \\
0
\end{array}\right)
$$

and $x^{\mathrm{T}}=\left(x_{1}^{\mathrm{T}} x_{2}^{\mathrm{T}} \cdots x_{m}^{\mathrm{T}}\right)$. Here, $x_{i}, i \in S_{m}$, is the state vector of vehicle $i$ (typically containing distance or distance error, velocity, acceleration, and possibly additional variables), with $S_{m}=\{i \in \mathbb{N} \mid 1 \leq i \leq m\}$ denoting the set of all vehicles in a platoon of length $m \in \mathbb{N}$. $u_{1}$ is the external input, which, in this case, is the input of an uncontrolled lead vehicle. $A_{0}$ and $B_{0}$ are the system matrix and the input matrix, respectively, of this lead vehicle, whereas $\tilde{A}_{0}$ and $\tilde{A}_{1}$ are the system and "input" matrices of the controlled follower vehicles. In addition, consider linear output functions according to

$$
y_{i}=C_{i} x, \quad i \in S_{m}
$$

where $y_{i}$ is the output of vehicle $i$, and $C_{i}$ is the corresponding output matrix. The Model (1) and (2), which will be further detailed in Section II-B, is considered $\mathcal{L}_{p}$ string stable if all outputs $y_{i}$ are bounded in the $\mathcal{L}_{p}$ sense for a bounded input $u_{1}$ and bounded initial condition perturbations $x(0)$, with $m \rightarrow \infty$, i.e., infinite string length. Hence, $y_{i}(t)$ must be bounded for all $i \in \mathbb{N}$ and for all $t \geq 0$. If, in addition

$$
\left\|y_{i}(t)-C_{i} \bar{x}\right\|_{\mathcal{L}_{p}} \leq\left\|y_{i-1}(t)-C_{i-1} \bar{x}\right\|_{\mathcal{L}_{p}}, \forall i \in \mathbb{N} \backslash\{1\}
$$

where $\bar{x}$ denotes the equilibrium state of (1) with $u_{1} \equiv 0$, and $\|\cdot\|_{\mathcal{L}_{p}}$ denotes the signal $p$-norm, ${ }^{1}$ the interconnected system is said to be strictly $\mathcal{L}_{p}$ string stable. For linear homogeneous cascaded systems with a unidirectional coupling and with a scalar input $u_{1}$ and scalar outputs $y_{i}$, the notions of $\mathcal{L}_{p}$ string stability and strict $\mathcal{L}_{p}$ string stability are equivalent [16].

Reformulating (1) and (2) in the Laplace domain, while exclusively focusing on input-output behavior, yields

$$
y_{i}(s)=P_{i}(s) u_{1}(s), \quad i \in S_{m}
$$

where $y_{i}(s)$ and $u_{1}(s), s \in \mathbb{C}$, denote the Laplace transforms of $y_{i}(t)$ and $u_{1}(t)$, respectively, and $P_{i}(s)=C_{i}(s I-A)^{-1} B$. Assuming that the system (4) is square and nonsingular, i.e., $P_{i}^{-1}(s)$ exists for all $i \in S_{m}$, the string stability complementary sensitivity (SSCS) is defined according to

$$
\Gamma_{i}(s):=P_{i}(s) P_{i-1}^{-1}(s)
$$

such that

$$
y_{i}(s)=\Gamma_{i}(s) y_{i-1}(s)
$$

Adopting the $\mathcal{L}_{2}$ signal norm (i.e., $p=2$ ), the following condition for strict $\mathcal{L}_{2}$ string stability holds [16].

Condition 1 (Strict $\mathcal{L}_{2}$ String Stability): The system (1) and (2), with Laplace-domain representation (4), is strictly $\mathcal{L}_{2}$ string stable if and only if

$$
\begin{aligned}
& \left\|P_{1}(s)\right\|_{\mathcal{H}_{\infty}}<\infty \\
& \left\|\Gamma_{i}(s)\right\|_{\mathcal{H}_{\infty}} \leq 1, \quad \forall i \in \mathbb{N} \backslash\{1\}
\end{aligned}
$$

where $\Gamma_{i}(s)$ is the SSCS according to (5), and $\|\cdot\|_{H_{\infty}}$ denotes the $H_{\infty}$ system norm.

\footnotetext{
${ }^{1}$ The signal $p$-norm or $\mathcal{L}_{p}$ norm of a vector $z(t)$ with elements $z_{k}(t)$ is defined as $\|z(t)\|_{\mathcal{L}_{p}}:=\left(\int_{-\infty}^{\infty} \sum_{k}\left|z_{k}(t)\right|^{p} \mathrm{~d} t\right)^{1 / p}$.
} 


\section{B. Cooperative Adaptive Cruise Control}

Based on earlier work on the control of interconnected vehicle strings, initiated in [17] and, among others, continued in [18], the concept of wireless-communication-based platooning has been introduced in the early 1990's [19]. This research resulted, among other things, in control strategies that are referred to as CACC. The objective of CACC is to regulate the intervehicle distances $d_{i}, i \in S_{m} \backslash\{1\}$, to a (small) desired value, while guaranteeing string stability. To briefly introduce a CACC controller that has the ability to satisfy this objective, consider the following model of a vehicle within a platoon of $m$ vehicles, as shown in Fig. 1, described by

$$
\begin{aligned}
& \left(\begin{array}{c}
\dot{v}_{1} \\
\dot{a}_{1}
\end{array}\right)=\left(\begin{array}{c}
a_{1} \\
-\frac{1}{\tau} a_{1}+\frac{1}{\tau} u_{1}
\end{array}\right) \\
& \left(\begin{array}{c}
\dot{d}_{i} \\
\dot{v}_{i} \\
\dot{a}_{i}
\end{array}\right)=\left(\begin{array}{c}
v_{i-1}-v_{i} \\
a_{i} \\
-\frac{1}{\tau} a_{i}+\frac{1}{\tau} u_{i}
\end{array}\right), \quad i \in S_{m} \backslash\{1\} .
\end{aligned}
$$

Here, $d_{i}=q_{i-1}-q_{i}-L_{i}$ is the distance between vehicle $i$ and $i-1$, where $q_{i}$ and $q_{i-1}$ are the rear bumper position of vehicle $i$ and $i-1$, respectively, and $L_{i}$ is the length of vehicle $i ; v_{i}$ is the velocity, and $a_{i}$ is the acceleration of vehicle $i$. This model, in fact, assumes that the vehicles are equipped with a low-level acceleration controller, which regulates the vehicle acceleration $a_{i}$ to the input $u_{i}$. Hence, the input $u_{i}$ should be interpreted as the desired acceleration, whereas the time constant $\tau$ represents the dynamics of the acceleration-controlled vehicle. In [12], it is shown that (8) adequately describes the longitudinal dynamics of the acceleration-controlled vehicles as used for the experimental validation (see Section VI).

Next, the following spacing policy is adopted:

$$
d_{\mathrm{r}, i}(t)=r_{i}+h v_{i}(t), \quad i \in S_{m} \backslash\{1\}
$$

where $d_{\mathrm{r}, i}$ is the desired distance between vehicle $i$ and $i-1$, $h$ is the time gap, and $r_{i}$ is the standstill distance. The main objective is to regulate the distance error

$$
e_{i}(t)=d_{i}(t)-d_{\mathrm{r}, i}(t), \quad i \in S_{m} \backslash\{1\}
$$

to zero, i.e.,

$$
a_{1}(t)=0 \forall t \geq 0 \Rightarrow \lim _{t \rightarrow \infty} e_{i}(t)=0 \forall i \in S_{m} \backslash\{1\}
$$

taking into account that this objective is, in general, only satisfied if the lead vehicle drives with a constant velocity, i.e., $a_{1}=0$. The following dynamic controller achieves this vehiclefollowing objective [12]:

$$
\dot{u}_{i}=-\frac{1}{h} u_{i}+\frac{1}{h}\left(k_{\mathrm{p}} e_{i}+k_{\mathrm{d}} \dot{e}_{i}+k_{\mathrm{dd}} \ddot{e}_{i}\right)+\frac{1}{h} u_{i-1}
$$

for all $i \in S_{m} \backslash\{1\}$, where $k_{\mathrm{p}}, k_{\mathrm{d}}$, and $k_{\mathrm{dd}}$ are the controller coefficients, and $h$ is the time gap as in (9).

Based on the vehicle model (8)-(10) and the controller (12), the state-space model of the controlled vehicle platoon can be formulated as in (1), with states $x_{i}^{\mathrm{T}}=\left(\begin{array}{llll}e_{i} & v_{i} & a_{i} & u_{i}\end{array}\right), i \in$

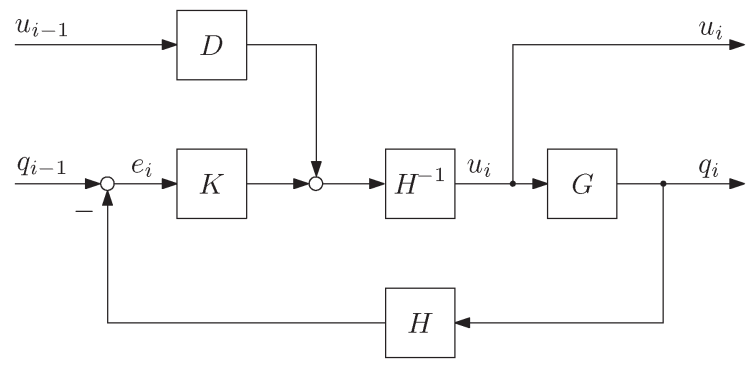

Fig. 2. Block scheme of the CACC system.

$S_{m}$ [16]. However, since the string stability conditions (7) are formulated in the Laplace domain, the model of the controlled vehicle platoon is also formulated in the Laplace domain. This finally leads to the block diagram of the closed-loop system for vehicle $i$ as shown in Fig. 2, with

$$
\begin{aligned}
& G(s)=\frac{q_{i}(s)}{u_{i}(s)}=\frac{1}{s^{2}(\tau s+1)} e^{-\phi s} \\
& H(s)=h s+1 \\
& K(s)=k_{\mathrm{p}}+k_{\mathrm{d}} s+k_{\mathrm{dd}} s^{2} \\
& D(s)=e^{-\theta s} .
\end{aligned}
$$

Here, $q_{i}(s)$ and $u_{i}(s)$ are the Laplace transforms of the vehicle position $q_{i}(t)$ and the desired acceleration $u_{i}(t)$, respectively; the vehicle transfer function $G(s)$ follows from $\ddot{q}_{i}=a_{i}$ and $\dot{a}_{i}=-(1 / \tau) a_{i}+(1 / \tau) u_{i}$ [see (8)], with an additional (driveline) delay $\phi$ as experimentally identified [12]. The spacing policy transfer function $H(s)$ is related to (9), and the controller $K(s)$ represents the error feedback in (12). Moreover, $\theta$ is the time delay induced by the wireless network. Note that, without loss of generality, $r_{i}=L_{i}=0 \forall i \in S_{m} \backslash\{1\}$ is assumed here.

Let the vehicle acceleration be taken as a basis for string stability, i.e., $y_{i}=a_{i}, \forall i \in S_{m}$, since it is physically relevant on the one hand and satisfies the requirement on $P_{1}(s)$ in Condition 1 on the other. The latter can be easily understood, because, with this choice of outputs, $P_{1}(s)=(1 /(\tau s+1)) e^{-\phi s}$; hence, $\left\|P_{1}(j \omega)\right\|_{H_{\infty}}=1$. The SSCS is then given by

$$
\Gamma_{\mathrm{CACC}}(s)=\frac{a_{i}(s)}{a_{i-1}(s)}=\frac{1}{H(s)} \frac{G(s) K(s)+D(s)}{1+G(s) K(s)}
$$

where $a_{i}(s)$ and $a_{i-1}(s)$ are the Laplace transforms of $a_{i}(t)$ and $a_{i-1}(t)$, respectively. It is noted that the SSCS (14) would be the same in case the velocity $v_{i}$ is chosen as output, since $a_{i}(s) /$ $a_{i-1}(s)=\left(s v_{i}(s)\right) /\left(s v_{i-1}(s)\right)=v_{i}(s) / v_{i-1}(s)$, but that the first requirement in Condition 1 would not be satisfied in that case. In addition, it is worth mentioning that the SSCS is independent of the vehicle index $i$, which is a direct consequence of the homogeneity assumption. Omitting the feedforward path yields an ACC controller, the $\operatorname{SSCS} \Gamma_{\mathrm{ACC}}(s)$ of which can be easily obtained from (14) with $D(s)=0$, yielding

$$
\Gamma_{\mathrm{ACC}}(s)=\frac{1}{H(s)} \frac{G(s) K(s)}{1+G(s) K(s)} .
$$




\section{GRaceful Degradation}

The difference of the CACC proposed in the previous section with its ACC counterpart is in the feedforward path (see Fig. 2), which includes the effect of the preceding vehicle's input $u_{i-1}$ into the control loop. This feedforward path is implemented through wireless intervehicle communication. Consequently, if the wireless link fails (or when the preceding vehicle is not equipped with CACC), CACC would degrade to ACC, leading to a significant increase in the minimum string-stable time gap. To implement an alternative fallback scenario that more gracefully degrades the CACC functionality, it is proposed to estimate the actual acceleration $a_{i-1}$ of the preceding vehicle, which can then be used as a replacement of the desired acceleration $u_{i-1}$ in case no communication updates are received. To further detail this approach, Section III-A first describes the target vehicle acceleration estimation, after which Section III-B incorporates the estimation algorithm into the $\mathrm{CACC}$ controller.

\section{A. Acceleration Estimation}

To describe an object's longitudinal motion, the Singer acceleration model [20] is adopted, being a reasonable choice for the formulation of the longitudinal vehicle dynamics. Note that rigourous analysis of longitudinal vehicle behavior in everyday traffic, and the dynamic vehicle model(s) as a result thereof, may lead to other choices; this is, however, outside the scope of this paper. The Singer acceleration model is defined by the following linear time-invariant system:

$$
\dot{a}(t)=-\alpha a(t)+u(t)
$$

with $a$ being the acceleration of the object vehicle and $u$ being the model input. $\alpha$ is equal to the inverse of the so-called maneuver time constant $\tau_{m}$, the choice of which will be briefly exemplified at the end of Section IV. The input $u$ is chosen as a zero-mean uncorrelated random process (i.e., white noise) to represent the unknown effects that may cause an object vehicle to accelerate or decelerate. To determine the variance of $u$, the object vehicle is assumed to exhibit a maximum acceleration $a_{\max }$ or deceleration $-a_{\max }$ with a probability $P_{\max }$, and to have a probability $P_{0}$ of zero acceleration, whereas other acceleration values are uniformly distributed. This results in the probability density function $p(a)$ as shown in Fig. 3, which appears to provide a satisfactory representation of the object's instantaneous maneuver characteristics [20]. Consequently, the object acceleration variance $\sigma_{a}^{2}$ is equal to

$$
\sigma_{a}^{2}=\frac{a_{\max }^{2}}{3}\left(1+4 P_{\max }-P_{0}\right) .
$$

It is shown in [20] that, in order to satisfy $p(a)$, the covariance $C_{u u}(\tau)$ of the white noise input $u$ in (16) reads

$$
C_{u u}(\tau)=2 \alpha \sigma_{a}^{2} \delta(\tau)
$$

where $\delta$ is the unit impulse function. As a result, the random variable $a$, satisfying a probability density function $p(a)$ with variance $\sigma_{a}^{2}$, while being correlated in time through the maneuver time constant $\tau_{m}$, is described as a random process $a(t)$,

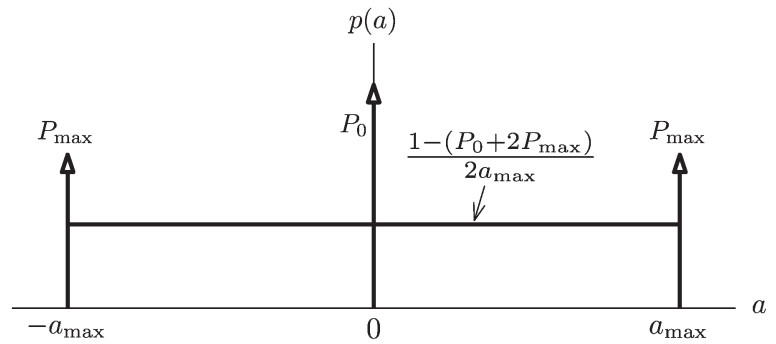

Fig. 3. Probability density function $p(a)$ of the object acceleration $a$.

being the output of a first-order system (16) with a white noise input $u(t)$ satisfying (18).

Using the acceleration model (16), the corresponding equation of motion can be described in the state space as

$$
\begin{aligned}
\dot{x}(t) & =A_{\mathrm{a}} x(t)+B_{\mathrm{a}} u(t) \\
y(t) & =C_{\mathrm{a}} x(t)
\end{aligned}
$$

where $x^{T}=\left(\begin{array}{lll}q & v & a\end{array}\right)$, with $q$ and $v$ being the object vehicle's position and velocity, respectively. The vector $y^{T}=(q v)$ is the output of the model, and the matrices $A_{\mathrm{a}}, B_{\mathrm{a}}$, and $C_{\mathrm{a}}$ are defined as

$$
A_{\mathrm{a}}=\left(\begin{array}{ccc}
0 & 1 & 0 \\
0 & 0 & 1 \\
0 & 0 & -\alpha
\end{array}\right), B_{\mathrm{a}}=\left(\begin{array}{l}
0 \\
0 \\
1
\end{array}\right), C_{\mathrm{a}}=\left(\begin{array}{lll}
1 & 0 & 0 \\
0 & 1 & 0
\end{array}\right) .
$$

Note that the state equation (19a) closely resembles the vehicle dynamics model (8) when replacing $\alpha$ by $\tau^{-1}$.

The model (19) is used as a basis for the estimation of the object vehicle acceleration by means of a Kalman filter [21]. To design this observer, ${ }^{2}$ the state-space model (19) is extended so as to include a process noise term $w(t)$, representing model uncertainty, and a measurement noise term $v(t)$, yielding

$$
\begin{aligned}
& \dot{x}(t)=A_{\mathrm{a}} x(t)+w(t) \\
& y(t)=C_{\mathrm{a}} x(t)+v(t) .
\end{aligned}
$$

The input $u(t)$ in (19a), which was assumed to be white noise, is included in (21) by choosing $w(t)=B_{\mathrm{a}} u(t)$, adopting the socalled equivalent noise approach [22]. $v(t)$ is a white noise signal with covariance matrix $R=E\left\{v(t) v^{\mathrm{T}}(t)\right\}$, as determined by the noise parameters of the onboard sensor used in the implementation of the observer, which, in this case, is a radar (see Section VI). Furthermore, using (18), the continuous-time process noise covariance matrix $Q=E\left\{w(t) w^{\mathrm{T}}(t)\right\}$ is equal to

$$
Q=B_{\mathrm{a}} E\left\{u(t) u^{\mathrm{T}}(t)\right\} B_{\mathrm{a}}^{\mathrm{T}}=\left(\begin{array}{ccc}
0 & 0 & 0 \\
0 & 0 & 0 \\
0 & 0 & 2 \alpha \sigma_{a}^{2}
\end{array}\right) .
$$

With the given $Q$ and $R$ matrices, the following continuoustime observer is obtained:

$$
\dot{\hat{x}}(t)=A_{\mathrm{a}} \hat{x}(t)+L_{\mathrm{a}}\left(y(t)-C_{\mathrm{a}} \hat{x}(t)\right)
$$

\footnotetext{
${ }^{2}$ Although for real-time implementation in the vehicle control computer a discrete-time Kalman filter is required, a continuous-time approach is adopted here, which simplifies the upcoming string stability analysis in Section IV.
} 
where $\hat{x}$ is the estimate of the object vehicle state $x^{\mathrm{T}}=(q v a)$, $L_{\mathrm{a}}$ is the continuous-time Kalman filter gain matrix, and $y$ is the measurement vector, consisting of position $q$ and velocity $v$ of the object vehicle. This observer provides a basis for the design of the fallback control strategy, as explained in the following section.

\section{B. CACC Fallback Scenario}

The fallback CACC strategy, which is hereafter referred to as "degraded CACC" (dCACC), aims to use the observer (23) to estimate the acceleration $a_{i-1}$ of the preceding vehicle at time instance $t$, taking into account sensor measurements up to time $t$. However, the measurement $y$ in (23), containing the absolute object position and velocity, is not available. Instead, the onboard sensor of the follower vehicle provides distance and relative velocity. Consequently, the estimation algorithm needs to be adapted, as described below.

As a first step, the observer (23) is described in the Laplace domain by a transfer function $T(s)$, which takes the actual position $q_{i-1}$ and velocity $v_{i-1}$ of the preceding vehicle, contained in the measurement vector $y$ in (23), as input. The output of $T(s)$ is the estimate $\hat{a}_{i-1}$ of the preceding vehicle's acceleration, being the third element of the estimated state. This yields the estimator

$$
\hat{a}_{i-1}(s)=T(s)\left(\begin{array}{c}
q_{i-1}(s) \\
v_{i-1}(s)
\end{array}\right)
$$

where $\hat{a}_{i-1}(s)$ denotes the Laplace transform of $\hat{a}_{i-1}(t)$, and $q_{i-1}(s)$ and $v_{i-1}(s)$ are the Laplace transforms of $q_{i-1}(t)$ and $v_{i-1}(t)$, respectively. Moreover, the $1 \times 2$ estimator transfer function $T(s)$ is equal to

$$
T(s)=\hat{C}(s I-\hat{A})^{-1} \hat{B}
$$

with

$$
\hat{A}=A_{\mathrm{a}}-L_{\mathrm{a}} C_{\mathrm{a}}, \hat{B}=L_{\mathrm{a}}, \hat{C}=\left(\begin{array}{lll}
0 & 0 & 1
\end{array}\right) .
$$

Note that $T(s)$ does not depend on vehicle index $i$ due to the homogeneity assumption.

The second step involves a transformation to relative coordinates, using the fact that (with $L_{i}=0$ )

$$
\begin{aligned}
& q_{i-1}(s)=d_{i}(s)+q_{i}(s) \\
& v_{i-1}(s)=\Delta v_{i}(s)+v_{i}(s)
\end{aligned}
$$

where $\Delta v_{i}(s)$ denotes the Laplace transform of the relative velocity $\Delta v_{i}(t)=\dot{d}_{i}(t)$. Substituting (27) into (24) yields

$$
\hat{a}_{i-1}(s)=T(s)\left(\begin{array}{c}
d_{i}(s) \\
\Delta v_{i}(s)
\end{array}\right)+T(s)\left(\begin{array}{c}
q_{i}(s) \\
v_{i}(s)
\end{array}\right) .
$$

As a result, the acceleration estimator is, in fact, split into a relative-coordinate estimator, i.e.,

$$
\widehat{\Delta a}_{i}(s):=T(s)\left(\begin{array}{c}
d_{i}(s) \\
\Delta v_{i}(s)
\end{array}\right)
$$

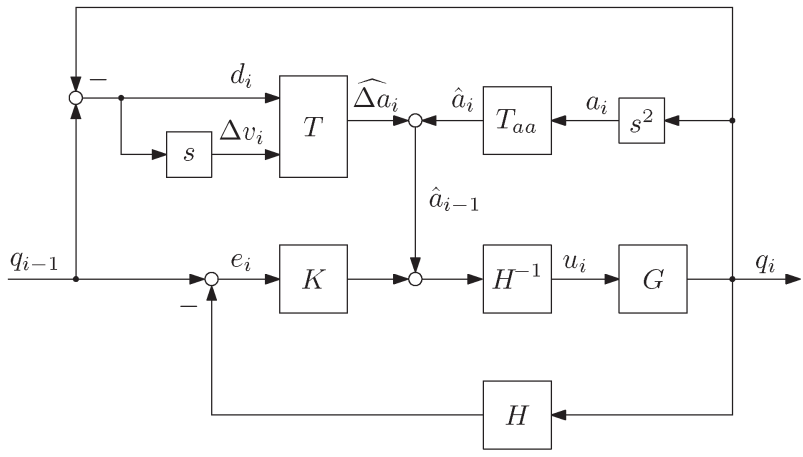

Fig. 4. Block scheme of the fallback dCACC system.

where $\widehat{\Delta a}_{i}(s)$ can be regarded as the Laplace transform of the estimated relative acceleration $\widehat{\Delta a}_{i}(t)$, and an absolutecoordinate estimator, i.e.,

$$
\hat{a}_{i}(s)=T(s)\left(\begin{array}{l}
q_{i}(s) \\
v_{i}(s)
\end{array}\right)
$$

where $\hat{a}_{i}(s)$ is the Laplace transform of the estimated local acceleration $\hat{a}_{i}(t)$.

Finally, $\hat{a}_{i}(s)$ in (30) can be easily computed with

$$
\begin{aligned}
\hat{a}_{i}(s) & =T(s)\left(\begin{array}{c}
q_{i}(s) \\
v_{i}(s)
\end{array}\right)=:\left(T_{a q}(s) \quad T_{a v}(s)\right)\left(\begin{array}{l}
q_{i}(s) \\
v_{i}(s)
\end{array}\right) \\
& =\left(\frac{T_{a q}(s)}{s^{2}}+\frac{T_{a v}(s)}{s}\right) a_{i}(s)=: T_{a a}(s) a_{i}(s)
\end{aligned}
$$

exploiting the fact that the local position $q_{i}(t)$ and velocity $v_{i}(t)$ are the result of integration of the locally measured acceleration $a_{i}(t)$, thereby avoiding the use of a potentially inaccurate absolute position measurement by means of a global positioning system. The transfer function $T_{a a}(s)$ acts as a filter for the measured acceleration $a_{i}$, yielding the "estimated" acceleration $\hat{a}_{i}$. In other words, the local vehicle acceleration measurement $a_{i}$ is synchronized with the estimated relative acceleration $\widehat{\Delta a_{i}}$ by taking the observer phase lag of the latter into account.

The control law of the fallback dCACC system is now obtained by replacing the preceding vehicle's input $u_{i-1}$ in the controller (12) with the estimated acceleration $\hat{a}_{i-1}$. As a result, the control law is formulated in the Laplace domain as

$$
u_{i}(s)=H^{-1}(s) \cdot\left\{K(s) e_{i}(s)+T(s)\left(\begin{array}{c}
d_{i}(s) \\
\Delta v_{i}(s)
\end{array}\right)+T_{a a}(s) a_{i}(s)\right\}
$$

which can be implemented using the radar measurement of the distance $d_{i}$ and the relative velocity $\Delta v_{i}$, and the locally measured acceleration $a_{i}$ and velocity $v_{i}$, the latter being required to calculate the distance error $e_{i}$ according to (9) and (10). The corresponding block diagram of the closed-loop dCACC system as a result of this approach is shown in Fig. 4, which can be compared with Fig. 2, showing the CACC scheme.

\section{String Stability of Degraded CACC}

To analyze the dCACC string stability properties, the output of interest is chosen to be the acceleration, since this directly 


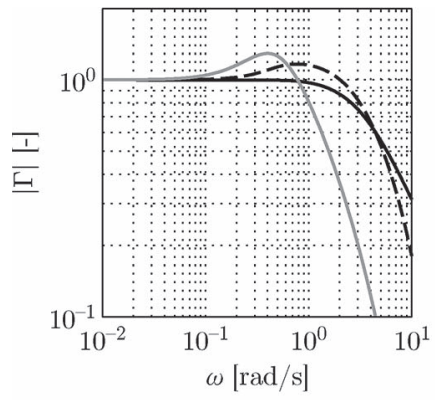

(a)

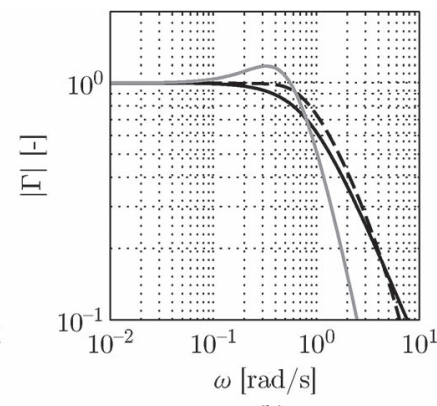

(b)
Fig. 5. SSCS frequency response magnitude in case of (solid black) CACC, (dashed black) dCACC, and (gray) ACC with (a) $h=0.3 \mathrm{~s}$ and (b) $h=1.3 \mathrm{~s}$.

TABLE I

VEHICLE AND CONTROLLER PARAMETERS

\begin{tabular}{l|l|l}
\hline \hline Symbol & Value & Description \\
\hline$\theta$ & $0.02 \mathrm{~s}$ & Communication delay \\
$\tau$ & $0.1 \mathrm{~s}$ & Vehicle time constant \\
$\phi$ & $0.2 \mathrm{~s}$ & Vehicle internal time delay \\
$k_{\mathrm{p}}$ & 0.2 & Controller gain (proportional) \\
$k_{\mathrm{d}}$ & 0.7 & Controller gain (differential) \\
$k_{\mathrm{dd}}$ & 0 & Controller gain \\
$a_{\max }$ & $3 \mathrm{~m} / \mathrm{s}^{2}$ & Maximum acceleration \\
$P_{\max }$ & 0.01 & Probability of maximum acceleration \\
$P_{0}$ & 0.1 & Probability of zero acceleration \\
$\alpha$ & $1.25 \mathrm{~s}^{-1}$ & Reciprocal maneuver time constant $\left(1 / \tau_{m}\right)$ \\
$\sigma_{d}^{2}$ & $0.029 \mathrm{~m}^{2}$ & Variance of measured distance \\
$\sigma_{\Delta v}^{2}$ & $0.017 \mathrm{~m}^{2} / \mathrm{s}^{2}$ & Variance of measured relative velocity \\
\hline \hline
\end{tabular}

guarantees the existence of $\left\|P_{1}(s)\right\|_{H_{\infty}}$, which is the first requirement in Condition 1 for strict $\mathcal{L}_{2}$ string stability. The $\operatorname{SSCS} \Gamma_{\mathrm{dCACC}}(s)$, as defined in (5), can then be computed using (6), with $y_{j}(s)=a_{j}(s), j=i, i-1$. As a result, with the closed-loop configuration given in Fig. 4, the following SSCS is obtained:

$$
\Gamma_{\mathrm{dCACC}}(s)=\frac{1}{H(s)} \frac{G(s)\left(K(s)+s^{2} T_{a a}(s)\right)}{1+G(s) K(s)} .
$$

The platoon of vehicles is string $\operatorname{stable}^{3}$ if also the second requirement as mentioned under Condition 1 holds, i.e., $\left\|\Gamma_{\mathrm{dCACC}}(s)\right\|_{H_{\infty}} \leq 1$. Furthermore, if the system is string unstable, $\left\|\Gamma_{\mathrm{dCACC}}(s)\right\|_{H_{\infty}}$ will exceed 1; still, in that case, we would aim at making this norm as low as possible to minimize disturbance amplification. The frequency response magnitudes $\left|\Gamma_{\mathrm{CACC}}(j \omega)\right|$ from (14), $\left|\Gamma_{\mathrm{dCACC}}(j \omega)\right|$ from (33), and $\left|\Gamma_{\mathrm{ACC}}(j \omega)\right|$ from (15), as a function of the frequency $\omega$, are shown in Fig. 5(a) and (b) for $h=0.3 \mathrm{~s}$ and $h=1.3 \mathrm{~s}$, respectively. Here, the model parameters, as summarized in Table I, are set according to the parameters of the test vehicles (see Section VI). From the frequency response magnitudes, it follows that for $h=0.3 \mathrm{~s}$, only CACC results in string-stable behavior, whereas for $h=1.3 \mathrm{~s}$, both CACC and dCACC yield string stability. Clearly, ACC is not string stable in either case.

\footnotetext{
${ }^{3}$ Recall that strict $\mathcal{L}_{2}$ string stability is equivalent to $\mathcal{L}_{2}$ string stability for the current system. Moreover, since only $\mathcal{L}_{2}$ string stability is considered, this notion will be simply referred to as string stability.
}
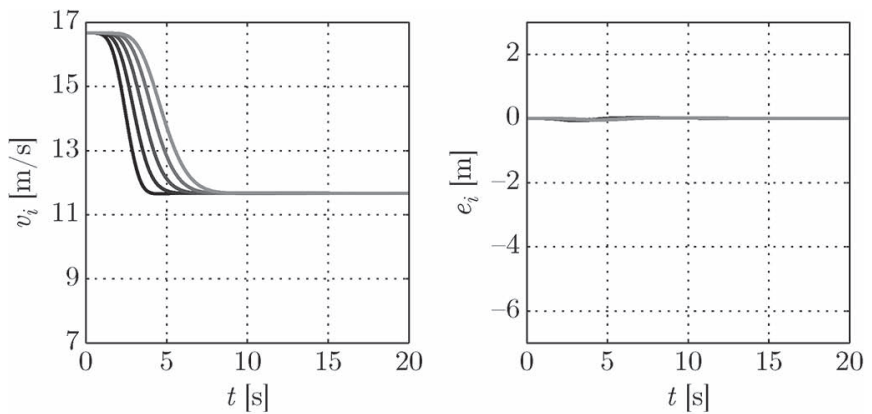

(a)
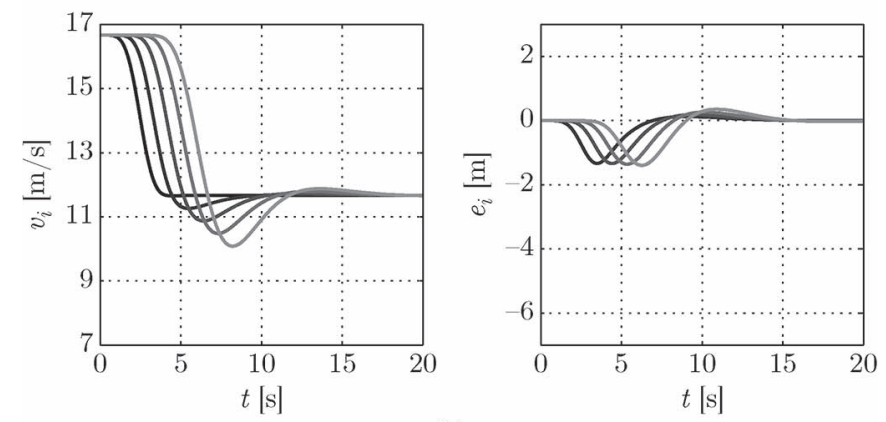

(b)
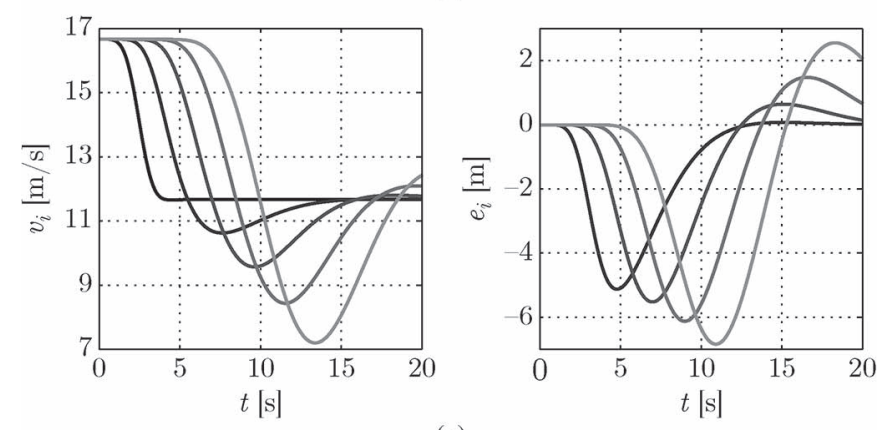

(c)

Fig. 6. Response of the velocity $v_{i}(t)$ (left column; black-light gray: $i=$ $1,2, \ldots, 5)$ and the distance error $e_{i}(t)$ (right column; black-light gray: $i=2$, $3,4,5$ ) for (a) CACC, (b) dCACC, and (c) ACC.

In addition to the frequency response functions, Fig. 6 shows time-domain responses. In this figure, the (velocity controlled) lead vehicle in a platoon of five vehicles follows a smooth down-step velocity profile, whereas the follower vehicles are controlled by either CACC, dCACC, or ACC, with $h=0.6 \mathrm{~s}$. As a result of this disturbance, the three systems respond very differently. From the velocity responses, it directly follows that the CACC system is string stable, whereas the dCACC and ACC systems start to propagate a shockwave. However, dCACC clearly outperforms ACC in terms of damping. The same effect can be seen in the responses of the distance error (10) in Fig. 6. Nevertheless, all systems show asymptotic tracking behavior, since the distance errors all converge to zero. For the given model and controller parameters, the stringstable time-gap region for dCACC appears to be $h \geq 1.23 \mathrm{~s}$, whereas for CACC and ACC, this appears to be $h \geq 0.25 \mathrm{~s}$ and $h \geq 3.16 \mathrm{~s}$, respectively. Consequently, dCACC represents a significant improvement over ACC regarding the minimum string-stable time gap.

The quality of the acceleration estimation as employed in dCACC can be illustrated as follows. For the same simulation 


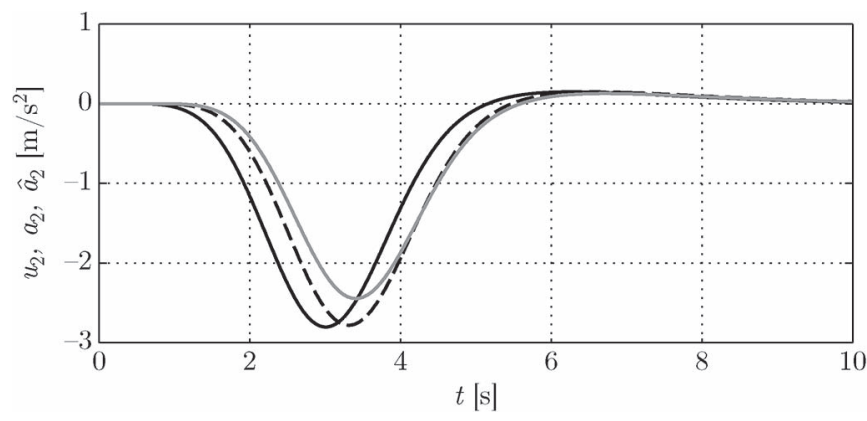

Fig. 7. Vehicle 2 acceleration: (solid black) desired acceleration $u_{2}$, (dashed black) actual acceleration $a_{2}$, and (gray) estimated acceleration $\hat{a}_{2}$.

as shown in Fig. 6, Fig. 7 shows the desired acceleration $u_{2}$ and the actual acceleration $a_{2}$ of vehicle $i=2$, as well as the acceleration $\hat{a}_{2}$ that is estimated by the follower vehicle $i=3$. As can be seen in the figure, $\hat{a}_{2}$, in itself providing a satisfactory estimation of $a_{2}$, shows a noticeable phase lag with respect to $u_{2}$, which is essentially the reason for the degraded string stability performance of dCACC.

The reciprocal maneuver time constant $\alpha=1.25$ of the Singer model can be increased so as to further reduce the frequency response peak of the SSCS function. However, it should be noted that there exists a tradeoff between the optimal value of $\alpha$ in view of string stability and the value needed for an acceptable level of ride comfort. As a rule of thumb, $0.5 \leq \alpha \leq 1.5$ appears to maintain both requirements at an acceptable level.

\section{Switching Criterion for Degraded CACC}

Until now, either full wireless communication under nominal conditions or a persistent loss of communication has been considered. However, in practice, the loss of the wireless link is often preceded by increasing communication latency [represented by the time delay $\theta$ in (13d)]. Intuitively, it can be expected that above a certain maximum allowable latency, wireless communication is no longer effective, upon which switching from CACC to dCACC is beneficial in view of string stability. This section proves this intuition to be true and also calculates the exact switching value for the latency, thereby providing a criterion for activation of dCACC.

From analysis of $\Gamma_{\mathrm{CACC}}$ in (14), it follows that the magnitude of the transfer function $(G K+D) /(1+G K)$ shows a peak value greater than 1 for a delay $\theta>0$. This peak value is suppressed by the remaining transfer function $1 / H=1 /(h s+$ $1)$ in $\Gamma_{\mathrm{CACC}}$, the effect of which is smaller for decreasing values of the time gap $h$, i.e., increasing cutoff frequency of $1 / H$. Consequently, for CACC, a minimum string-stable time gap $h_{\min , \mathrm{CACC}}$ must exist, which depends on the delay $\theta$. Along the same line of thought, it can be shown that for dCACC, a minimum string-stable time gap also exists (obviously independent of the communication delay), which appears to be $h_{\mathrm{min}, \mathrm{dCACC}}=1.23 \mathrm{~s}$, as already mentioned in the previous section.

Fig. 8 shows $h_{\min , \mathrm{CACC}}$ as a function of $\theta$ and $h_{\min , \mathrm{dCACC}}$. Here, $h_{\min , \mathrm{CACC}}(\theta)$ has been obtained by searching for the smallest $h$ for each $\theta$, such that $\left\|\Gamma_{\mathrm{CACC}}(s)\right\|_{H_{\infty}}=1$. This

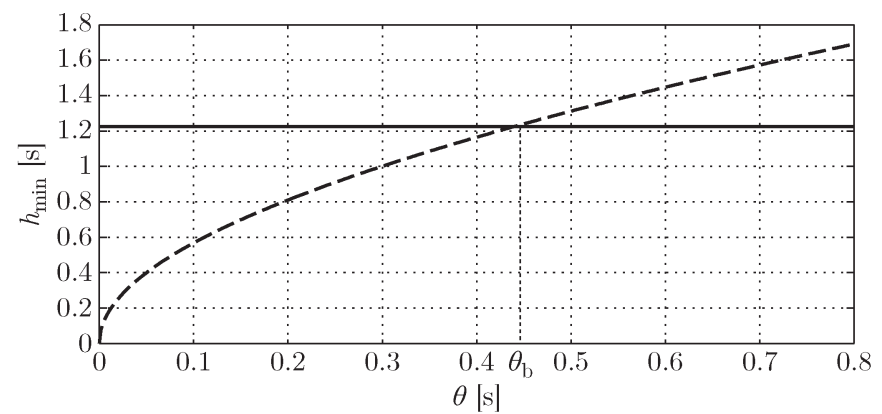

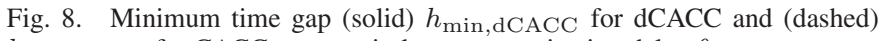
$h_{\mathrm{min}, \mathrm{CACC}}$ for CACC versus wireless communication delay $\theta$.

figure clearly shows a breakeven point $\theta_{\mathrm{b}}$ of the delay $\theta$, i.e., $h_{\text {min, }, \mathrm{dCACC}}=h_{\min , \mathrm{CACC}}\left(\theta_{\mathrm{b}}\right)$, which is equal to $\theta_{\mathrm{b}}=0.44 \mathrm{~s}$ for the current controller and acceleration observer. The figure also indicates that for $\theta<\theta_{\mathrm{b}}$, it is beneficial to use CACC in view of string stability, since this allows for smaller time gaps, whereas for $\theta>\theta_{\mathrm{b}}$, dCACC is preferred. This is an important result, since it provides a criterion for switching from CACC to $\mathrm{dCACC}$ and vice versa in the event that there is not (yet) a total loss of communication, although it would require monitoring the communication time delay when CACC is operational.

As a final remark on this matter, it should be noted that the above analysis only holds for a communication delay that slowly varies, compared with the system dynamics. Moreover, it does not cover the situation in which data samples (packets) are intermittently lost, rather than delayed. These effects require further analysis, to which end [8] and [10] provide a number of tools.

\section{EXPERIMENTAL VALIDATION}

The CACC system, with the added graceful degradation feature, is implemented in three identical passenger cars (Toyota Prius III Executive), equipped with a wireless communication device that follows the ITS G5 standard [23], enabling the vehicles to communicate control-related information such as the desired acceleration $u_{i}$. The relative position of the preceding vehicle and its relative velocity are measured by a long-range radar, which is an original vehicle component in this case. Furthermore, a real-time platform executes the CACC with a sampling time $t_{\mathrm{s}}=0.01 \mathrm{~s}$, yielding the desired vehicle acceleration $u_{i}$, which is then forwarded to a low-level acceleration controller of the vehicle. This section first presents experimentally obtained frequency responses to validate the string stability properties of dCACC, compared with those of CACC and ACC, after which measured time responses are shown to validate the performance of the controllers in general.

\section{A. Frequency Response Experiments}

The frequency response tests are conducted with two vehicles only. Here, the lead vehicle is velocity controlled, with a reference velocity profile $v_{\mathrm{r}}(t)$. This test signal should provide sufficient frequency content for performing nonparametric system identification, particularly to identify the SSCS function in the relevant frequency range. Toward this end, a random-phase 


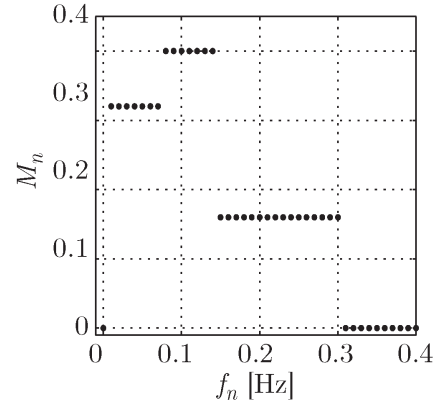

(a)

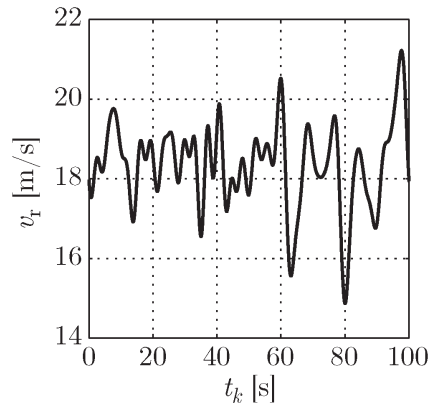

(b)
Fig. 9. Velocity-reference test signal used for identification of the controlled vehicle platoon: (a) frequency-domain magnitude $M_{n}$ and (b) corresponding time-domain signal $v_{\mathrm{r}}(k)$.

multisine signal is selected, which covers the frequency range $f \in[0,0.3] \mathrm{Hz}([0,1.9] \mathrm{rad} / \mathrm{s})$. This frequency range is chosen so as to include the maximum SSCS magnitudes. The test signal is designed in the frequency domain by choosing frequency weightings $M_{n}$, with $n=0,1, \ldots,(N / 2)-1$ and $N$ being the number of frequency intervals up to the sampling frequency $f_{\mathrm{s}}=1 / t_{\mathrm{s}}$. The chosen frequency-domain magnitudes $M_{n}$ of the test signal, as a function of the discrete frequency $f_{n}=n \Delta f$, with frequency interval $\Delta f=f_{\mathrm{s}} / N$, are shown in Fig. 9(a); the resulting discrete-time signal $v_{\mathrm{r}}(k)$ at time $t_{k}=k t_{\mathrm{s}}$ with $k=0,1, \ldots, N-1$, is shown in Fig. 9(b).

To run the dCACC system in the two test vehicles, the relative-acceleration estimator in (28) has been implemented in the follower vehicle $(i=2)$ using the discrete-time equivalent of the filter (23), with measurement input vector

$$
y(k)=\left(\begin{array}{ll}
d_{2}(k) & \Delta v_{2}(k)
\end{array}\right)^{\mathrm{T}}
$$

being the radar output, and with the state vector

$$
\hat{x}(k)=\left(\begin{array}{lll}
\hat{d}_{2}(k) & \widehat{\Delta v}_{2}(k) & \widehat{\Delta a}_{2}(k)
\end{array}\right)^{\mathrm{T}} .
$$

This yields the estimated relative acceleration $\widehat{\Delta a} a_{2}(k)$, based on which the absolute lead vehicle acceleration $a_{1}(k)$ is estimated by adding the filtered locally measured acceleration $\hat{a}_{2}(k)$, using the discrete-frequency equivalent of $T_{a a}(s)$ in (31), combined with an onboard acceleration sensor.

Using the measured data from the tests, nonparametric system identification is performed to estimate the magnitude of the transfer function from $v_{1}$ to $v_{2}$, resulting in the estimated SSCS magnitudes for CACC, dCACC, and ACC, denoted by $\left|\hat{\Gamma}_{\mathrm{CACC}}\left(j \omega_{n}\right)\right|,\left|\hat{\Gamma}_{\mathrm{dCACC}}\left(j \omega_{n}\right)\right|$, and $\left|\hat{\Gamma}_{\mathrm{ACC}}\left(j \omega_{n}\right)\right|$, respectively, with $\omega_{n}=n 2 \pi \Delta f$. Subsequently, these are compared with the theoretical frequency response magnitudes $\left|\Gamma_{\mathrm{CACC}}(j \omega)\right|,\left|\Gamma_{\mathrm{dCACC}}(j \omega)\right|$, and $\left|\Gamma_{\mathrm{ACC}}(j \omega)\right|$, obtained through evaluation over the given frequency range of the SSCS transfer functions given in (14), (33), and (15), respectively, using the $h=0.6 \mathrm{~s}$ time gap. The result is shown in Fig. 10 . In this figure, it can be seen that the experimental results match with the theoretical ones in the frequency range of excitation as indicated in Fig. 9(a), i.e., for frequencies up to $1.9 \mathrm{rad} / \mathrm{s}=$ $0.3 \mathrm{~Hz}$. It should be noted, however, that a larger excitation frequency interval may have been chosen, to avoid the excessive

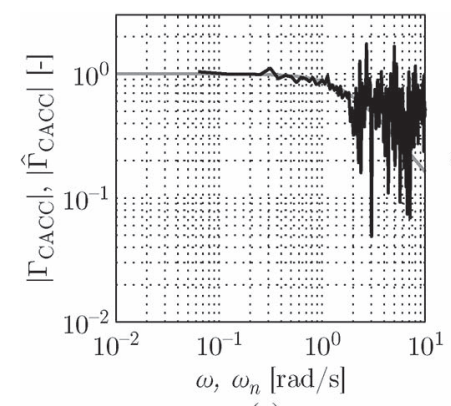

(a)

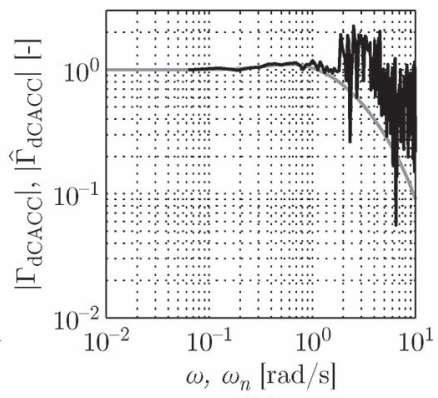

(b)

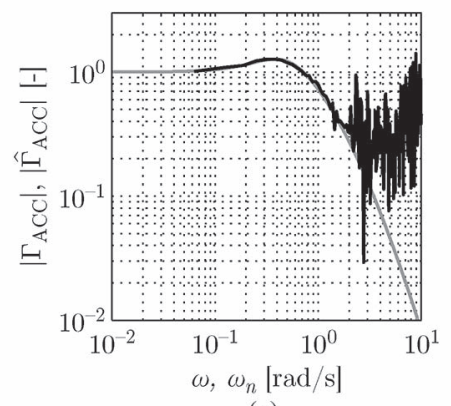

(c)

Fig. 10. (Black) Experimental SSCS frequency response magnitude $|\hat{\Gamma}|$ and (gray) the theoretical counterpart $|\Gamma|$ of the system subject to (a) CACC, (b) dCACC, and (c) ACC.

noise for $\omega>1.9 \mathrm{rad} / \mathrm{s}$ and, consequently, to obtain a better estimate for dCACC around the cutoff frequency. Nevertheless, it can be concluded that the experiments confirm the improvement with respect to string stability obtained with dCACC compared with the conventional ACC fallback scenario.

\section{B. Time Response Experiments}

The time response experiments are conducted with three vehicles and are identical to those shown in Fig. 6: Starting from a situation in which the platoon is in steady state at $16.67 \mathrm{~m} / \mathrm{s}(60 \mathrm{~km} / \mathrm{h})$ with $h=0.6 \mathrm{~s}$, the velocity controlled lead vehicle performs a smooth velocity step of $-5 \mathrm{~m} / \mathrm{s}$. The measured velocity responses and distance error responses are shown in Fig. 11. Comparing the velocity responses with the simulated responses in Fig. 6 directly reveals that the practical experiments are very similar to the theoretical results: CACC is again clearly string stable, whereas dCACC and ACC are not. Nevertheless, the amount of overshoot is much smaller with dCACC than with ACC. Note that also the magnitude of the velocity responses is very close to that of the simulations. The distance error responses slightly deviate from the simulated responses as far as the amplitude is concerned, but still clearly show the same trend, despite the rather large noise level, which is inherent to the distance measurement by the forwardlooking radar. As will be seen in the last experiment, explained hereafter, this measurement noise leads to noise in the estimated acceleration, which is why the velocity response in case of dCACC is less smooth, compared with CACC and ACC. As already mentioned at the end of Section IV, a smoother behavior (hence, improved ride comfort) can be obtained by decreasing the value of the reciprocal maneuver time constant $\alpha$, but at the cost of increasing the minimum string-stable time gap. 

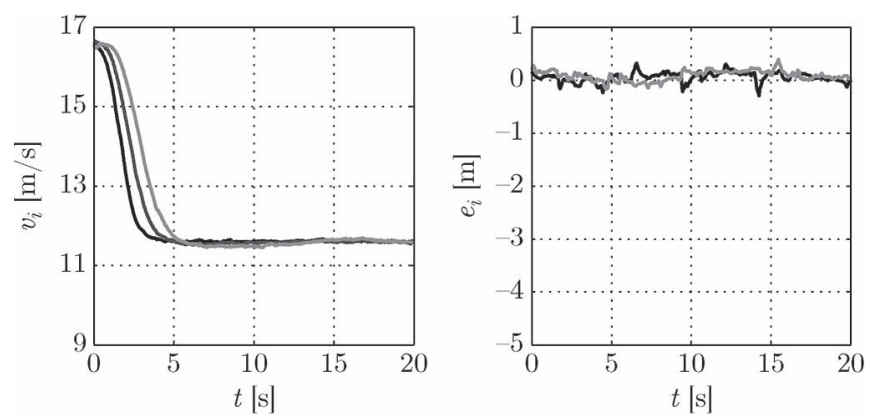

(a)
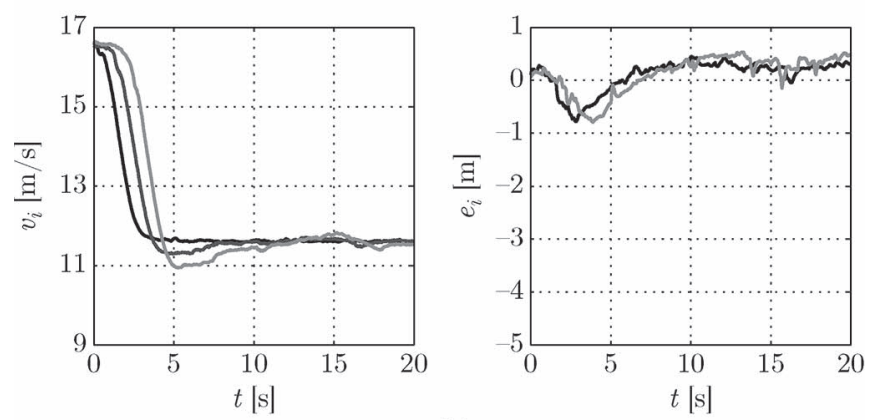

(b)
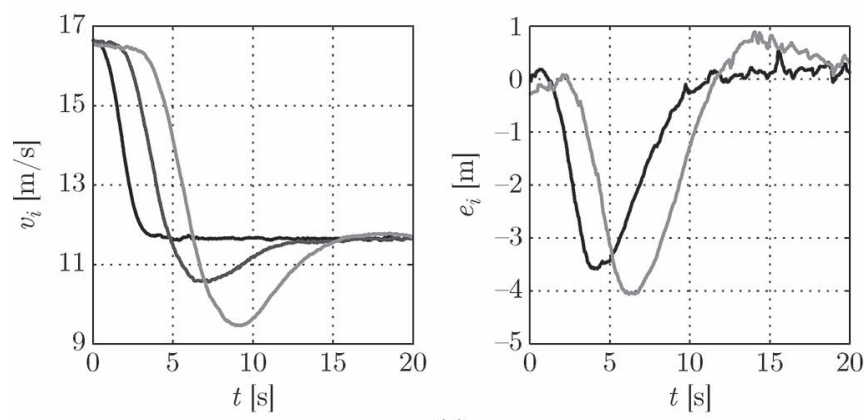

(c)

Fig. 11. Measured response of the velocity $v_{i}(t)$ (left column; black-light gray: $i=1,2,3$ ) and the distance error $e_{i}(t)$ (right column; black, light gray: $i=2,3$ ) for (a) CACC, (b) dCACC, and (c) ACC.

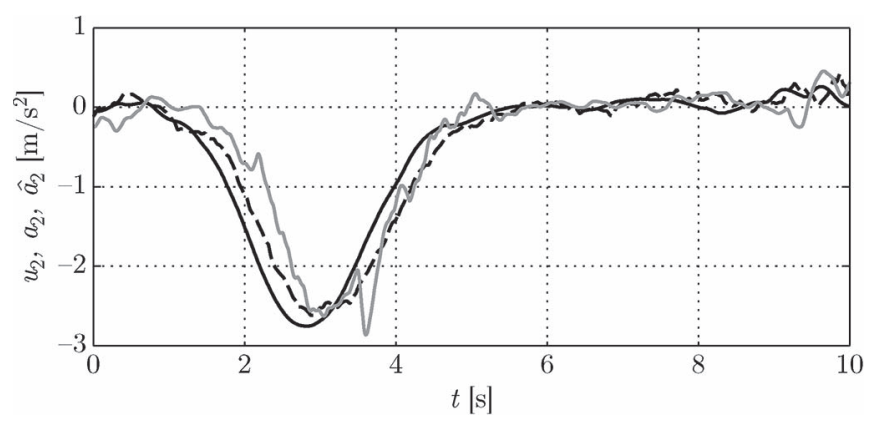

Fig. 12. (Solid black) Desired acceleration $u_{2}$, (dashed black) measured acceleration $a_{2}$, and (gray) estimated acceleration $\hat{a}_{2}$.

Finally, using the same experiment, Fig. 12 shows the desired acceleration $u_{2}(t)$ and the actual measured acceleration $a_{2}(t)$ of the first follower vehicle, both received in the last follower vehicle via the wireless link, as well as the estimated acceleration $\hat{a}_{2}(t)$, computed in the last follower vehicle. As can be seen in this figure, $\hat{a}_{2}(t)$ provides a satisfactory estimation of $a_{2}(t)$ but shows a significant phase lag with respect to $u_{2}(t)$, which corresponds to the simulation results as depicted in Fig. 7. As mentioned earlier, this phase lag accounts for the degraded string stability performance of dCACC. Furthermore, $\hat{a}_{2}(t)$ shows a considerable noise level due to the quality of the radar measurements. This behavior could be improved by tuning the value of the reciprocal maneuver time constant $\alpha$. Fortunately, the measurement noise is hardly noticeable in vehicle 3 , which uses $\hat{a}_{2}(t)$ as a feedforward signal, because the precompensator $H^{-1}(s)$ together with the vehicle dynamics act as a series connection of first-order lowpass filters with time constant $h$ and $\tau$, respectively [see (13) and Fig. 4].

\section{CONCLUSION}

To accelerate practical implementation of CACC in everyday traffic, wireless communication faults must be taken into account. To this end, a graceful degradation technique for CACC was presented, serving as an alternative fallback scenario to ACC. The idea behind the proposed approach is to obtain the minimum loss of functionality of CACC when the wireless link fails or when the preceding vehicle is not equipped with wireless communication means. The proposed strategy, which is referred to as dCACC, uses an estimation of the preceding vehicle's current acceleration as a replacement to the desired acceleration, which would normally be communicated over a wireless link for this type of CACC. In addition, a criterion for switching from CACC to dCACC was presented, in the case that wireless communication is not (yet) lost, but shows increased latency. It was shown that the performance, in terms of string stability of dCACC, can be maintained at a much higher level compared with an ACC fallback scenario. Both theoretical as well as experimental results showed that the dCACC system outperforms the ACC fallback scenario with respect to string stability characteristics by reducing the minimum string-stable time gap to less than half the required value in case of ACC.

\section{REFERENCES}

[1] R. Rajamani and C. Zhu, "Semi-autonomous adaptive cruise control systems," IEEE Trans. Veh. Technol., vol. 51, no. 5, pp. 1186-1192, Sep. 2002.

[2] P. Seiler, A. Pant, and K. Hedrick, "Disturbance propagation in vehicle strings," IEEE Trans. Autom. Control, vol. 49, no. 10, pp. 1835-1842, Oct. 2004.

[3] G. J. L. Naus, R. P. A. Vugts, J. Ploeg, M. J. G. van de Molengraft, and M. Steinbuch, "String-stable CACC design and experimental validation: A frequency-domain approach," IEEE Trans. Veh. Technol., vol. 59, no. 9, pp. 4268-4279, Nov. 2010.

[4] S. E. Shladover, D. Su, and X.-Y. Lu, "Impacts of cooperative adaptive cruise control on freeway traffic flow," in Proc. 91st TRB Апnи. Meet., Jan. 22-26, 2012, pp. 1-17.

[5] D. V. A. H. G. Swaroop, "String Stability of Interconnected Systems: An Application to Platooning in Automated Highway Systems," University of California, California Partners for Advanced Transit and Highways (PATH), Berkeley, CA, USA, UCB-ITS-PRR-97-14, Jan. 1997.

[6] X. Liu, A. Goldsmith, S. S. Mahal, and J. K. Hedrick, "Effects of communication delay on string stability in vehicle platoons," in Proc. IEEE Conf. Intell. Transp. Syst., Aug. 25-29, 2001, pp. 625-630.

[7] J. I. Ge and G. Orosz, "Dynamics of connected vehicle systems with delayed acceleration feedback," Transp. Res. Part C, Emerging Technol., vol. 46, pp. 46-64, Sep. 2014.

[8] S. Öncü, "String Stability of Interconnected Vehicles: Network-Aware Modelling, Analysis and Experiments," Ph.D. dissertation, Eindhoven Univ. Technol., Eindhoven, The Netherlands, Jan. 2014.

[9] D. Eckhoff, N. Sofray, and R. German, "A performance study of cooperative awareness in ETSI ITS G5 and IEEE WAVE," in Proc. 10th Annu. Conf. Wireless On-Demand Netw. Syst. Serv., Mar. 18-20, 2013, pp. 196-200. 
[10] P. Seiler and R. Sengupta, "An $H_{\infty}$ approach to networked control," IEEE Trans. Autom. Control, vol. 50, no. 3, pp. 356-364, Mar. 2005.

[11] C. Lei et al., "Evaluation of CACC string stability using SUMO, Simulink, and OMNeT++," EURASIP J. Wireless Commun. Netw., vol. 116, pp. 112, Mar. 2012

[12] J. Ploeg, B. T. M. Scheepers, E. van Nunen, N. van de Wouw, and H. Nijmeijer, "Design and experimental evaluation of cooperative adaptive cruise control," in Proc. 14th Int. IEEE Conf. Intell. Transp. Syst., Oct. 5-7, 2011, pp. 260-265.

[13] D. Swaroop and J. K. Hedrick, "String stability of interconnected systems," IEEE Trans. Autom. Control, vol. 41, no. 3, pp. 349-357, Mar. 1996.

[14] R. F. Curtain, O. V. Iftime, and H. J. Zwart, "System theoretic properties of a class of spatially invariant systems," Automatica, vol. 45, no. 7, pp. 1619-1627, Jul. 2009.

[15] X.-Y. Lu and J. K. Hedrick, "Practical string stability for longitudinal control of automated vehicles," Veh. Syst. Dyn., vol. 41, pp. 577-586, 2004.

[16] J. Ploeg, N. van de Wouw, and H. Nijmeijer, " $\mathcal{L}_{p}$ string stability of cascaded systems: Application to vehicle platooning," IEEE Trans. Control Syst. Technol., vol. 22, no. 2, pp. 786-793, Mar. 2014.

[17] W. S. Levine and M. Athans, "On the optimal error regulation of a string of moving vehicles," IEEE Trans. Autom. Control, vol. 11, no. 3, pp. 355361, Jul. 1966

[18] L. Peppard, "String stability of relative-motion PID vehicle control systems," IEEE Trans. Autom. Control, vol. 19, no. 5, pp. 579-581, Oct. 1974.

[19] S. Sheikholeslam and C. A. Desoer, "Longitudinal control of a platoon of vehicles," in Proc. Am. Control Conf., May 23-25, 1990, pp. 291-296.

[20] R. A. Singer, "Estimating optimal tracking filter performance for manned maneuvering targets," IEEE Trans. Aerosp. Electron. Syst., vol. AES-6, no. 4, pp. 473-483, Jul. 1970.

[21] P. S. Maybeck, Stochastic Models, Estimation, and Control, vol. 141. New York, NY, USA: Academic, 1979, ser. Mathematics in Science and Engineering.

[22] X. R. Li and V. P. Jilkov, "A survey of maneuvering target tracking. Part IV: Decision-based methods," in Proc. SPIE Conf. Signal Data Process. Small Targets, Apr. 2002, pp. 4728-4760.

[23] E. G. Ström, "On medium access and physical layer standards for cooperative intelligent transport systems in Europe," Proc. IEEE, vol. 99, no. 7, pp. 1183-1188, Jul. 2011.

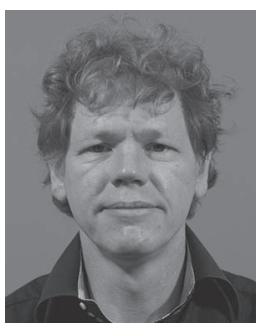

Jeroen Ploeg received the M.Sc. degree in mechanical engineering from Delft University of Technology, Delft, The Netherlands, in 1988 and the Ph.D. degree in mechanical engineering on the control of vehicle platoons from Eindhoven University of Technology, Eindhoven, The Netherlands, in 2014.

From 1989 to 1999 he was a Researcher with Koninklijke Hoogovens (currently Tata Steel), IJmuiden, The Netherlands, where his main interest was the dynamic process control of large-scale industrial plants. Since 1999 he has been a Senior Research Scientist with the Integrated Vehicle Safety Department, Netherlands Organization for Applied Scientific Research TNO, Helmond, The Netherlands. His interests include control system design for cooperative and automated vehicles in general and automated vehicle platoons in particular, and motion control of wheeled mobile robots.

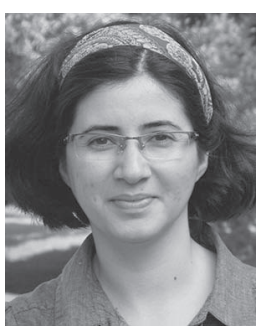

Elham Semsar-Kazerooni (M'05) received the B.Sc. degree (with distinction) from Shiraz University, Shiraz, Iran, in 2000; the M.Sc. degree (with distinction) from University of Tehran, Tehran, Iran, in 2003; and the Ph.D. degree from Concordia University, Montreal, QC, Canada, in 2009, all in electrical engineering.

From 2010 to 2012 she was an FQRNT Postdoctoral Fellow with University of Toronto, Toronto, ON, Canada. Since then she has been with the Integrated Vehicle Safety Department, Netherlands Organization for Applied Scientific Research TNO, Helmond, The Netherlands. Her research interests include cooperative control systems, control of vehicle platoons, consensus achievement, nonlinear systems analysis, and optimal system design.

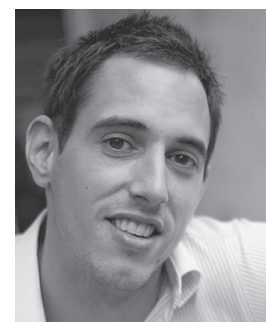

Guido Lijster received the B.Eng. degree in electrical engineering from HAN University of Applied Sciences, Arnhem, The Netherlands, in 2008 and the M.Sc. degree in mechanical engineering from Eindhoven University of Technology, Eindhoven, The Netherlands, in 2012

In 2011 he completed his Master's graduation project in the Integrated Vehicle Safety Department, Netherlands Organization for Applied Scientific Research TNO, Helmond, The Netherlands, where he worked on the performance of the cooperative adaptive cruise control system subject to unreliable wireless communication. Since 2013 he has been a Mechatronics Engineer with TSCC Technology, Eindhoven, supporting customers in the design of various industrial high-tech production machines for the solar industry.

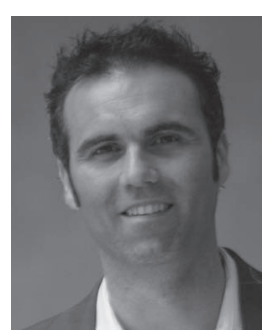

Nathan van de Wouw received the M.Sc. degree (with honors) and the Ph.D. degree in mechanical engineering from Eindhoven University of Technology, Eindhoven, The Netherlands, in 1994 and 1999, respectively.

From 1999 to 2014 he was with the Department of Mechanical Engineering, Eindhoven University of Technology, as an Assistant/Associate Professor. $\mathrm{He}$ is currently an Adjunct Full Professor with University of Minnesota, Minneapolis, MN, USA. In 2000 he was with Philips Applied Technologies, Eindhoven, and in 2001 he was with the Netherlands Organization for Applied Scientific Research TNO, Delft, The Netherlands. He was a Visiting Professor with University of California, Santa Barbara, CA, USA, during 2006-2007; University of Melbourne, Melbourne, Australia, during 2009-2010; and University of Minnesota during 2012-2013. He has published a large number of journal and conference papers and the books "Uniform Output Regulation of Nonlinear Systems: A Convergent Dynamics Approach," with A. V. Pavlov and H. Nijmeijer (Birkhauser, 2005) and "Stability and Convergence of Mechanical Systems with Unilateral Constraints," with R. I. Leine (Springer-Verlag, 2008). His research interests include the analysis and control of nonlinear/nonsmooth systems and networked control systems.

Dr. van de Wouw is an Associate Editor of Automatica and IEEE TRANSACTIONS ON CONTROL SYSTEMS TECHNOLOGY.

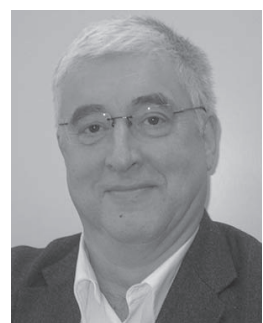

Henk Nijmeijer (F'00) received the M.Sc. and $\mathrm{Ph} . \mathrm{D}$. degrees in mathematics from University of Groningen, Groningen, The Netherlands, in 1979 and 1983, respectively.

From 1983 to 2000 he was with the Department of Applied Mathematics, University of Twente, Enschede, The Netherlands. Since 2000 he has been a Full Professor with Eindhoven University of Technology, Eindhoven, The Netherlands, chairing the Dynamics and Control group of the Department of Mechanical Engineering. He has published a large number of journal and conference papers, and several books, including "Nonlinear Dynamical Control Systems," with A. J. van der Schaft (Springer-Verlag, 1990); "Synchronization of Mechanical Systems," with A. Rodriguez (World Scientific, 2003); "Dynamics and Bifurcations of Non-Smooth Mechanical Systems," with R. I. Leine (Springer-Verlag, 2004); and "Uniform Output Regulation of Nonlinear Systems," with A. Pavlov and N. van de Wouw (Birkhauser, 2005).

Dr. Nijmeijer was the Editor-in-Chief of Journal of Applied Mathematics until 2009, the Corresponding Editor of the SIAM Journal on Control and Optimization, and a Board Member of International Journal of Control, Automatica, Journal of Dynamical Control Systems, International Journal of Bifurcation and Chaos, International Journal of Robust and Nonlinear Control, Journal of Nonlinear Dynamics, and Journal of Applied Mathematics and Computer Science. He was awarded the IET Heaviside Premium in 1990. In the 2008 research evaluation of the Dutch Mechanical Engineering Departments, the Dynamics and Control group was evaluated as excellent regarding all aspects (quality, productivity, relevance, and viability). 